\title{
COOL ENVELOPES OF POST-NOVAE \\ CONSTRAINTS ON THE DECLINE OF THE WHITE DWARF
}

\author{
Grażyna Stasińska \\ DAEC Observatoire de Paris-Meudon \\ 92195 - Meudon principal cedex , France \\ Romuald Tylenda \\ Copernicus Astronomical Center, Chopina 12/18 \\ 87-100 Torun', Poland
}

Some post-novae, e.g. DQ Her, CP Pup, T Aur, have cool envelopes, Te $<1000 \mathrm{~K}$. As has been shown by Ferland et al. (1984) this effect is primarily due to large overabundances of the CNO elements which at low densities can very effectively cool off the nebular gas through infra-red lines.

We have constructed an extensive grid of photoionization models in order to study the exact conditions required for the electron temperature to drop to such low values. As can be seen in Figs. 1 to 4 , our results can be summarized as follows.

1 - The abundance of oxygen which is the dominant coolant in the conditions considered has to be at least 10 times the solar value (with $\mathrm{C}$ and $\mathrm{N}$ accordingly overabundant).

2 - The gas density, $n$, has to be below $1000 \mathrm{~cm}^{-3}$.

3 - The effective temperature of the ionizing source, $T_{\text {eff, }}$, has to be below $210^{5} \mathrm{~K}$.

4 - The ionization parameter $U=Q_{H} /\left(4 \pi r^{2} n c\right)\left(Q_{H}\right.$ : number of ionizing photons emitted per second by the central source, $r$ : radius of the envelope) has to be between a certain range, typically $10^{-2}<U<10^{-3}$, whose exact limits, however, depend on other parameters such as $T_{\text {eff }}$ and the abundances.

We have carried out a detailed analysis of these conditions in the case of the envelope of $D Q$ Her. Using the observed value of the electron temperature $T_{e} \approx 500 \mathrm{~K}$ and the observed Hell $4686 / \mathrm{H} \beta$ ratio of 0.2 , and adopting the distance of $420 \mathrm{pc}$ (determined from the expansion parallax), we have found that, for a wide range of effective temperatures, densities and abundances, the constraints on $U$ imply that the luminosity of the ionizing source in $\mathrm{QD}$ Her had to be below $10 \mathrm{~L}_{\odot}$ at the time of the observations. In other words, the white dwarf in DQ Her had to decrease in luminosity by at least 3 orders of magnitude 40 years after the nova outburst. This is an important constraint for the theoretical models of the evolution of the white dwarf after the outburst. If a grid of reliable 
full-cycle nova models is available, this constraint could be used to determine a lower limit to the white dwart mass.

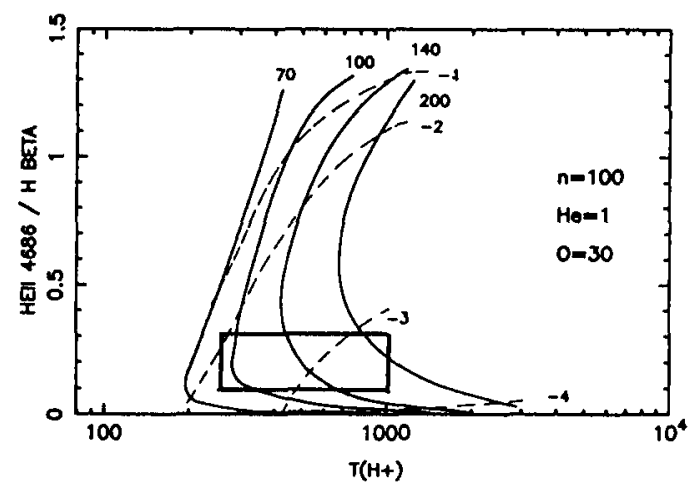

Fig. 1. The relation Hell $4686 / \mathrm{Hb}$ versus the mean electron temperature $T\left(\mathrm{H}^{+}\right)$for post-nova envelopes having $\mathrm{n}=100 \mathrm{~cm}^{-3}$ and abundances of $\mathrm{He}$, $C, N$, and $O$ equal to $1,50,250$ and 30 times solar, respectively. Full curves: models of given effective temperature of the ionizing star, i.e. $70,100,140$ and $200 \times 10^{3} \mathrm{~K}$. Dashed curves: loci of constant ionization parameter, i.e. $\log U=-1,-2,-3$ and -4 . The rectangle shows the observed values for the envelope of DQ Her, i.e. Hell $4686 / \mathrm{H} \beta=0.2 \pm 0.1$ and $\log T\left(\mathrm{H}^{+}\right)=2.7 \pm 0.3$.

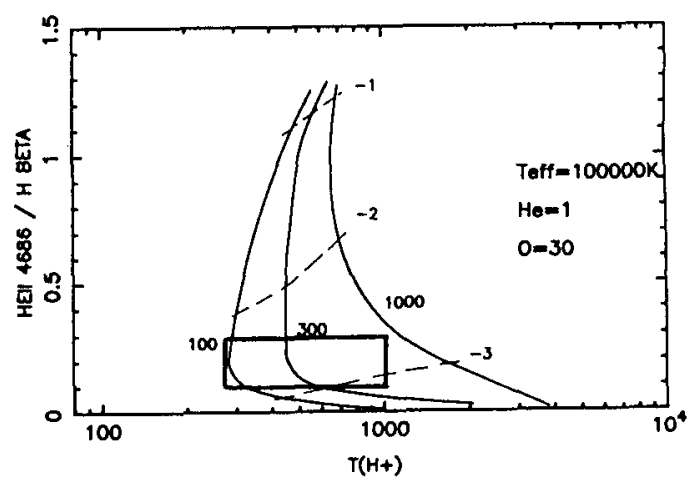

Fig. 3. The same as Fig. 1 but for different values of the nebular density, i.e. 100,300 and $1000 \mathrm{~cm}^{-3}$. Full curves: models with $T_{\text {eff }}=100 \times 10^{3} \mathrm{~K}$. Dashed curves: loci of constant value of the ionization parameter, i.e. $\log U=-1,-2,-3$.

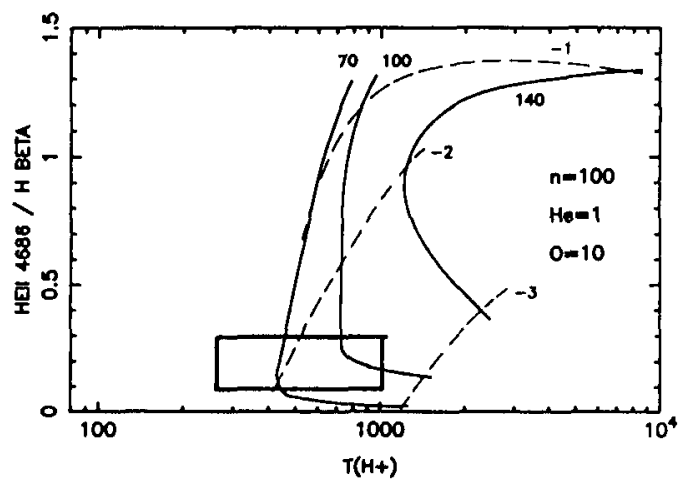

Fig. 2. The same as Fig. 1 but for lower abundances of $\mathrm{CNO}$, i.e. 15,80 and 10 times the solar values, respectively.

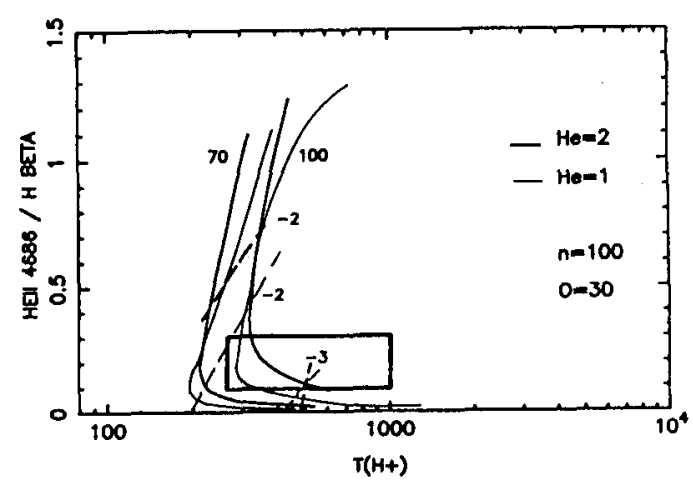

Fig. 4. The same as Fig. 1 but comparing two abundances of He, i.e. 1 (thin curves) and 2 (thick curves) times the solar values. Full curves are labelled with $T_{\text {eff, i.e. }} 70$ and $100 \times 10^{3} \mathrm{~K}$. Dashed curves show the loci of constant value of the ionization parameter, i.e. $\log U=-2,-3$.

\section{References:}

Ferland,G.J., Williams,R.E., Lambert,D.L., Shields,G.A., Slovak,M., Gondhalekar,P.M., Truran,J.W, 1984, Astrophys. J. 281,194. 